\title{
Correction to: Influence of dosimetry method on bone lesion absorbed dose estimates in PSMA therapy: application to mCRPC patients receiving Lu-177-PSMA-I\&T
}

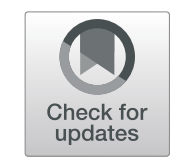

Julia Brosch-Lenz ${ }^{1 *}$, Carlos Uribe ${ }^{2,3}$, Astrid Gosewisch', Lena Kaiser' ${ }^{1}$ Andrei Todica1', Harun Illhan', Franz Josef Gildehaus ${ }^{1}$, Peter Bartenstein ${ }^{1}$, Arman Rahmim ${ }^{2,3,4}$, Anna Celler ${ }^{3}$, Sibylle Ziegler ${ }^{1}$ and Guido Böning ${ }^{1}$

The original article can be found online at https://doi.org/10.1186/ s40658-021-00369-4

* Correspondence: Julia. BroschLenz@med.uni-muenchen.de 'Department of Nuclear Medicine, University Hospital, LMU Munich, Marchioninistrasse 15, 81377 Munich, Germany

Full list of author information is available at the end of the article
Correction to: EJNMMI Phys 8, 26 (2021)

https://doi.org/10.1186/s40658-021-00369-4

Following publication of the original article [1], it was reported that due to a typesetting error some text was mistakenly introduced in the "MC method: Patient-specific Monte Carlo (MC) absorbed dose simulation" and "Comparison of dosimetry methods" subsections.

The erroneous text is highlighted in bold in the below passages and has been removed in the original article.

In the "MC method: Patient-specific Monte Carlo (MC) absorbed dose simulation" the affected sentence was:

A CT scan of a Gammex tissue characterization phantom (Gammex 467; Gammex Inc., Middleton, WI) using the same imaging parameters from the patient scans was perfMC method: Patient-specificormed, which confirmed the HU-to-density relationship of our CT device with that implemented in GATE. GATE converts HU-to-density values with internal tables based on Schneider et al. [22].

The corrected sentence reads:

A CT scan of a Gammex tissue characterization phantom (Gammex 467; Gammex Inc., Middleton, WI) using the same imaging parameters from the patient scans was performed, which confirmed the HU-to-density relationship of our CT device with that implemented in GATE. GATE converts HU-to-density values with internal tables based on Schneider et al. [22].

In the "Comparison of dosimetry methods" sub-section the affected sentence was:

(c) The Author(s). 2021 Open Access This article is licensed under a Creative Commons Attribution 4.0 International License, which permits use, sharing, adaptation, distribution and reproduction in any medium or format, as long as you give appropriate credit to the original author(s) and the source, provide a link to the Creative Commons licence, and indicate if changes were made. The images or other third party material in this article are included in the article's Creative Commons licence, unless indicated otherwise in a credit line to the material. If material is not included in the article's Creative Commons licence and your intended use is not permitted by statutory regulation or exceeds the permitted use, you will need to obtain permission directly from the copyright holder. To view a copy of this licence, visit http://creativecommons.org/licenses/by/4.0/. 
The additional density wePatient example showing the transversal slice ofighting of $V S V_{\text {weighted }}^{\text {soft }}$ and $V S V_{\text {weighted }}^{\text {soft }}$, led to an overall smaller range of percentage differences than the associated method without weighting.

The corrected sentence reads:

The additional density weighting of $V S V_{\text {weighted }}^{\text {soft }}$ and $V S V_{\text {weighted }}^{\text {soft }}$, led to an overall smaller range of percentage differences than the associated method without weighting.

The original article has been updated.

\section{Author details}

'Department of Nuclear Medicine, University Hospital, LMU Munich, Marchioninistrasse 15, 81377 Munich, Germany.

${ }^{2}$ PET Functional Imaging, BC Cancer, 600 West 10th Avenue, Vancouver, BC V5Z 4E6, Canada. ${ }^{3}$ Department of Radiology, University of British Columbia, 2775 Laurel Street, Vancouver, BC V5Z 1M9, Canada. ${ }^{4}$ Department of Integrative Oncology, BC Cancer Research Centre, 675 West 10th Avenue, Vancouver, BC V5Z 1L3, Canada.

Published online: 13 April 2021

\section{Reference}

1. Brosch-Lenz J, Uribe C, Gosewisch A, et al. Influence of dosimetry method on bone lesion absorbed dose estimates in PSMA therapy: application to mCRPC patients receiving Lu-177-PSMA-I\&T. EJNMMI Phys. 2021;8:26 https://doi.org/10.11 86/s40658-021-00369-4. 